\title{
Partial anomalous pulmonary venous connection and pulmonary arterial hypertension
}

\author{
SANDEep SAHAY, ${ }^{1}$ RICHARD A. KRASUSKIㄴ AND AdRIANo R. TONELLI ${ }^{3}$ \\ ${ }^{1}$ Department of Medicine, Akron General Medical Center, Akron, ${ }^{2}$ Adult Congenital Heart Disease Services, \\ Department of Cardiovascular Medicine, Heart and Vascular Institute, and ${ }^{3}$ Department of Pulmonary, Allergy, \\ and Critical Care Medicine, Respiratory Institute, Cleveland Clinic, Cleveland, Ohio, USA
}

\begin{abstract}
Background and objective: Isolated partial anomalous pulmonary venous connection (PAPVC) has been implicated as a cause of pulmonary arterial hypertension (PAH); however this condition is often overlooked in the diagnostic work up of patients with $\mathrm{PH}$. We studied the prevalence of PAH both in patients with isolated PAPVC or associated with other congenital heart diseases (CHD) such as atrial septal defect (ASD). We also aimed to identify factors related to the presence of PAH in these patients.

Methods: We retrospectively analyzed data from the Adult CHD database at the Cleveland Clinic, USA between October 2005-2010. We included all patients diagnosed with PAPVC with or without other CHD. We excluded all patients with previous corrective surgeries. Results: We identified $14(2.5 \%)$ patients with PAPVC. Group I included patients with PAPVC (with or without patent foramen ovale (PFO)). Group II included patients with PAPVC associated with other CHD. PAH was seen in six $(6 / 14,42.8 \%)$ patients, two $(2 / 7,28.5 \%)$ in group I and four $(4 / 7,57.1 \%)$ in group II $(P=0.3)$. The mean pulmonary artery pressure in all patients $(n=14)$ was $29.5 \pm 13.8 \mathrm{~mm} \mathrm{Hg}$. Group I had a mean PAP of $23.6 \pm 6.6 \mathrm{~mm} \mathrm{Hg}$ as compared to $33.7 \pm 16.5 \mathrm{~mm} \mathrm{Hg}$ for group II $(P=0.34)$. The two patients in group I with PAH had either two anomalous pulmonary veins or a condition (sickle cell disease) that could potentially explain the haemodynamic findings.

Conclusions: Patients with PAPVC (with or without PFO) in the absence of other CHD had normal pulmonary arterial pressure (PAP) unless they have two pulmonary veins with anomalous return or associated conditions known to cause PAH.
\end{abstract}

Key words: congenital heart disease, partial anomalous pulmonary venous connection, pulmonary arterial hypertension, atrial septal defect.

Correspondence: Sandeep Sahay, Akron General Medical Center, 400 Wabash Avenue, Akron, OH 44307, USA. Email: sandeepsahay@gmail.com

Received 7 November 2011; invited to revise 3 January 2012; revised 18 January 2012; accepted 23 February 2012 (Associate Editor: Neil Eves).

\section{SUMMARY AT A GLANCE}

Isolated partial anomalous pulmonary veins are often overlooked in the diagnostic work up of the pulmonary arterial hypertension. In the clinical practice, these cases are often labelled as primary pulmonary hypertension. Our case series highlights the development of pulmonary arterial hypertension in individuals with isolated partial anomalous pulmonary veins.

\section{INTRODUCTION}

Partial anomalous pulmonary venous connection (PAPVC) is found in around $0.4-0.7 \%$ of autopsies. ${ }^{1,2}$ In most cases, the anomalous venous connection involves the right pulmonary veins ${ }^{1,3}$ that drain into the superior vena cava (SVC) or the right atrium (RA). Anomalous pulmonary veins less frequently drain into the inferior vena cava (IVC), innominate vein or the coronary sinus. ${ }^{3}$ These anomalous veins are frequently associated with other congenital heart anomalies, predominantly atrial septal defects (ASD). An anomalous pulmonary venous connection occurs in approximately $10-15 \%$ of ostium secundum ASD and $85 \%$ of patients with sinus venosus ASD. ${ }^{4-6}$

PAPVC adversely affects the cardio-pulmonary physiology by presenting as a left-to-right shunt, aggravated by frequent coexistent atrial septal defects. ${ }^{7,8}$ In a normal person, each pulmonary vein contributes an average $25 \%$ of the total pulmonary blood flow. However, in anomalous pulmonary vein return, the shunt flow can be higher since the circulation is preferentially directed to the right side due to lower pressure in the RA and superior vena cava than in the left atrium. This effect becomes more pronounced in conditions that tend to increase left atrial pressure such as systemic hypertension or left heart disease. Depending on a variety of factors, some patients with PAPVC eventually develop right-sided volume overload that leads to pulmonary arterial hypertension $(\mathrm{PAH})$ and right heart failure. ${ }^{8}$ 
Although ASD is frequently diagnosed using transthoracic or transoesophageal echocardiography with the aid of agitated saline, PAPVC is often overlooked predominantly in cases not associated with other congenital heart diseases (CHD). A review of the literature reveals cases where PAPVC was missed in patients who underwent transcatheter closure of ASD. ${ }^{9,10}$ In these cases, the long-standing increased pulmonary blood flow could eventually lead to the development of the PAH. ${ }^{7}$ In our experience, it is not uncommon to see patients with PAH due to PAPVC diagnosed as idiopathic pulmonary arterial hypertension. This prompted us to conduct a retrospective review all the patients seen in our adult congenital heart disease clinic to identify those with PAPVC, in order to study the prevalence of PAH both in patients with isolated PAPVC or associated with other congenital heart diseases such as ASD.

\section{METHODS}

We retrospectively analyzed the Adult Congenital Heart Disease database at the Cleveland Clinic, Cleveland, Ohio. Cleveland Clinic is a tertiary level multidisciplinary hospital. We reviewed all enrolled patients from October 2005 to October 2010. The Institutional Review Board (IRB) at Cleveland Clinic approved the present study (protocol number 10-1129) and waived the need for informed consent. Our study aim was to look for the prevalence of PAH both in patients with isolated PAPVC or associated with other congenital heart diseases such as ASD and to identify factors related to the presence of PAH in these patients.

We included patients diagnosed with PAPVC with or without other congenital heart defects. We excluded individuals who had corrective heart surgeries as we were looking for the undiagnosed PAPVC in adulthood. We reviewed the clinical data available on these patients using our hospital electronic medical records. The patients were enrolled in the adult congenital heart disease database once the diagnosis of congenital heart disease was confirmed with appropriate investigations. Some patients were symptomatic upon enrolment, while others were asymptomatic and were diagnosed incidentally.

A total of 564 patients were enrolled in adult congenital heart disease database during the aforementioned period. Twenty-two (3.9\%) subjects had PAPVC and/or sinus venosus ASD. We included in our initial search sinus venosus ASD, as this condition is frequently associated with PAPVC. Of these 22 subjects, we selected 14 patients who had confirmed PAPVC and no previous corrective heart surgeries. We divided these patients into two groups. Group I included patients PAPVC with or without patent foramen ovale (PFO). Group II included patients with PAPVC associated with other congenital heart defects. We included patients with concomitant PFO in group I because this condition is a prevalent finding encountered in up to $25 \%$ of the general population, ${ }^{11}$ usually does not affect cardiopulmonary haemodynamics, and most patients with this lesion remain asymptomatic for life. Patients were diagnosed with PAPVC by pulmonary venous angiography, cardiac magnetic resonance imaging (MRI) or computed tomography (CT) scan. All these patients were further evaluated with right heart catheterization as a part of our protocol. We carefully reviewed the haemodynamic data obtained during right heart catheterization and defined pulmonary hypertension (PAH) as a mean pulmonary artery pressure of $25 \mathrm{~mm} \mathrm{Hg}$ or above with pulmonary artery occlusion pressure $\leq 15 \mathrm{~mm} \mathrm{Hg}$ and pulmonary vascular resistance $>3$ Wood units. ${ }^{12,13}$

\section{Statistical analysis}

Continuous variables were expressed as mean and standard deviation when normally distributed. Twogroup comparison was performed by Mann-Whitney $U$-test. Comparisons between categorical data were performed by Fischer's exact test. All the $P$-values reported are two-tailed. A $P$-value of $<0.05$ was considered significant. The statistical analyses were performed using the statistical package SPSS, version 17 (SPSS Inc; Chicago, IL).

\section{RESULTS}

\section{Prevalence of PAPVC}

Over the period of five years (2005-2010), we identified $14(2.5 \%)$ patients with PAPVC and no previous corrective cardiac surgeries. Each group had seven patients. Three patients in group I had PFO along with PAPVC, while four had PAPVC alone (Table 1). Group II included patients with PAPVC associated with other congenital heart defects (Table 2). Group II included seven patients of which six had PAPVC associated with sinus venosus ASD, and one individual had PAPVC associated with ostium secundum ASD. The mean age and gender in groups I and II are described in Table 3 . In group I, five patients were asymptomatic at the time of diagnosis, while the other two had dyspnea of six-month and indeterminate duration, respectively. In group II, three patients were clinically asymptomatic (two had cardiac murmur on clinical examination) at the time of diagnosis, while the rest had dyspnoea as their main symptom, with a mean duration of symptoms before diagnosis of 2.2 years. One patient in group II was diagnosed by the aberrant placement of the Hickman catheter noticed on chest X-ray (Table 2).

\section{Pulmonary arterial hypertension (PAH)}

Pulmonary arterial hypertension ${ }^{12}$ was seen in six $(6 / 14,42.8 \%)$ patients, two $(2 / 7,28.5 \%)$ in group I and four $(4 / 7,57.1 \%)$ in group II $(P=0.3)$. In group I, patients with PAH either had two pulmonary veins with anomalous return (right upper pulmonary vein drained in the superior vena cava and left upper pulmonary vein drained in the left brachiocephalic vein) 


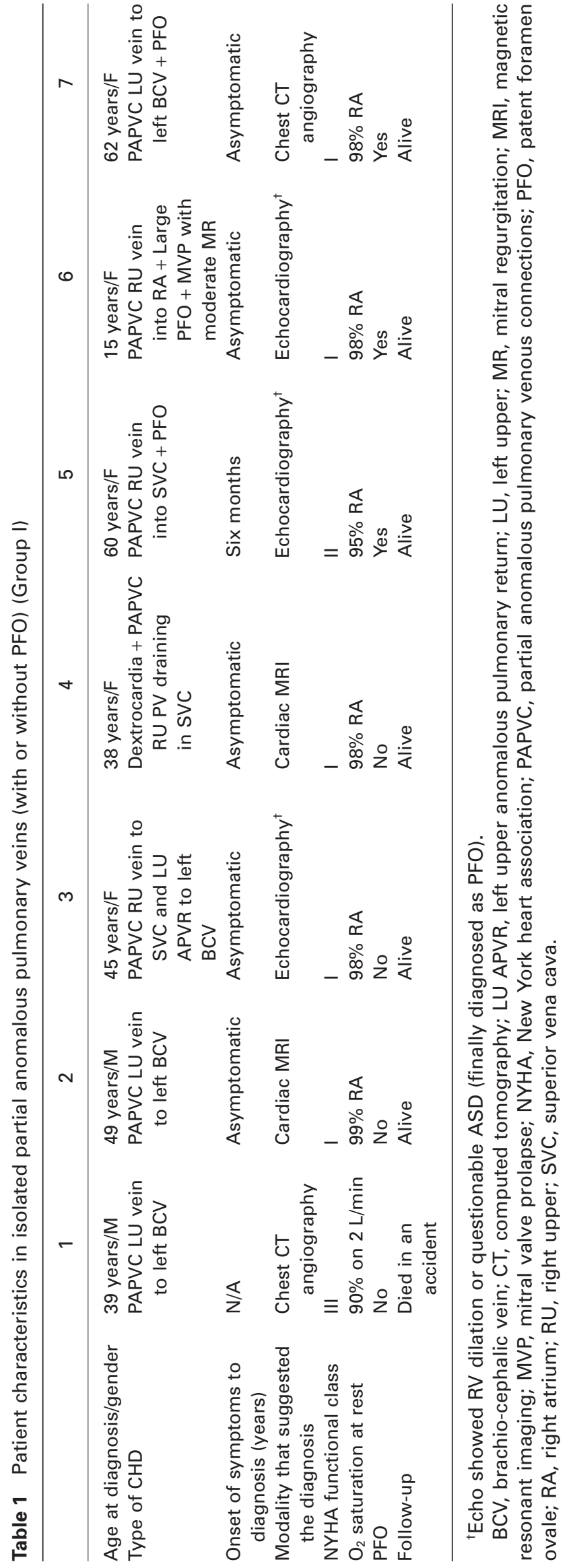

or an associated condition known to cause PAH such as haemoglobin sickle cell (SC) disease (Table 1). Except for the patient with haemoglobin SC disease, no other subject either in group I or II had associated diseases known to cause PAH. The overall mean pulmonary arterial pressure (PAP) in all patients $(n=14)$ was $29.5 \pm 13.8$ (range 20-32) $\mathrm{mm} \mathrm{Hg}$. There was no statistical difference the mean PAP when compared between the groups I and II. (Table 3). The mean PAP in the five patients of group I, who had one anomalous PAPVC and no associated conditions known to cause $\mathrm{PAH}$, was $20 \pm 6.0 \mathrm{~mm} \mathrm{Hg}(P=0.25$ when compared to group II). The mean Qp : Qs, available in 12 patients (11 patients had Qp : Qs measured by RHC and 1 by MRI), was $2.6 \pm 1.3$ (range 1.3-3). There was no significant difference in the Qp:Qs between groups I and II (Table 3). No association was found between Qp : Qs with either mean PAP or right ventricular systolic pressure (RVSP) on linear regression analysis. Coronary arteries were studied in 12 patients and were found to be normal.

\section{Echocardiographic data}

Echocardiography was performed in all patients as a part of their evaluation. In group I, the right ventricle (RV) was mild or moderately dilated in two patients and severely dilated in one. In group II, mild RV dilatation was seen in two patients, moderate RV dilatation in four and severe RV dilatation in one. Right ventricular function was normal in all patients in group I, except one. In group II, one patient had normal RV function, two had mild, three had moderate and one had severe RV dysfunction. The overall RVSP in 14 patients was $42.8 \pm 18.5$ (range 29.5-50) $\mathrm{mm} \mathrm{Hg}$. The RVSP in group I was $34.6 \pm 9.2 \mathrm{~mm} \mathrm{Hg}$, while in group 2 was $51.0 \pm 22.0 \mathrm{~mm} \mathrm{Hg}(P=0.16)$.

\section{Follow-up}

Among all $(n=14)$, seven patients underwent correction of their congenital heart defects. In group I, only one patient (first patient in Table 1) had redirection surgery of the anomalous vein to the left atrium, while the rest of the patients were clinically followed without the need for adding PAH-targeted therapies. In group II, six of the seven patients underwent surgical correction of the congenital defects (Table 2). There was no significant difference in the preoperative and the immediate post-operative RVSP $(54.8 \pm 27.2$ vs $43 \pm 19.5 \mathrm{~mm} \mathrm{Hg}, P=0.4)$. Of patients that underwent corrective surgery for the congenital heart defects (mean follow up of 30.6 months, range 1-55 months), one died from a non-medical related accident. In patients that did not undergo corrective surgery, one died, while awaiting lung transplantation, due to Eisenmenger's syndrome and right heart failure (patient number 7 in Table 2).

\section{DISCUSSION}

Partial anomalous pulmonary venous connection was first demonstrated during cardiac catheterization 


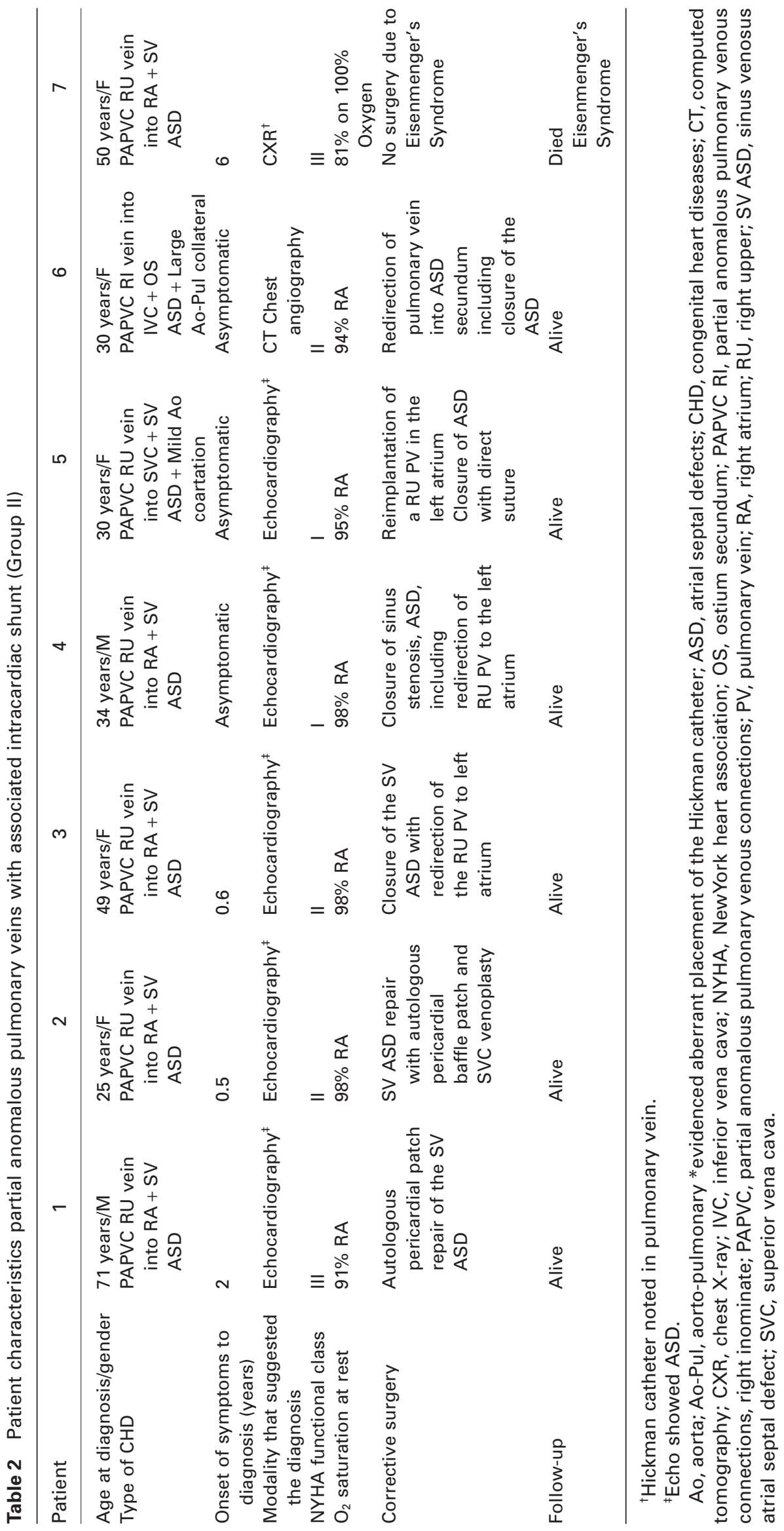


Table 3 Comparison of the haemodynamic variables observed during right heart catheterization in group I and group II partial anomalous pulmonary venous return

\begin{tabular}{lccc}
\hline & $\begin{array}{c}\text { Group I }(n=7) \\
\text { Mean } \pm \text { SD or } \%\end{array}$ & $\begin{array}{c}\text { Group II }(n=7) \\
\text { Mean } \pm \text { SD or } \%\end{array}$ & $\begin{array}{c}\text { P-value (Mann-Whitney } \\
\text { or Fischer exact test) }\end{array}$ \\
\hline Age (years) & $44.8 \pm 19.2$ & $41.3 \pm 16.2$ & 0.62 \\
Gender (female) \% & $71 \%$ & $71 \%$ & 1 \\
NYHA functional class & 1.4 & 2.0 & 0.21 \\
Systolic ABP (mm Hg) & $125 \pm 22.1$ & $122.1 \pm 13.8$ & 0.88 \\
Diastolic ABP (mm Hg) & $75.2 \pm 10.8$ & $72 \pm 8.8$ & 0.53 \\
HR (bpm) & $84.4 \pm 10.7$ & $81.6 \pm 12.4$ & 0.88 \\
RA pressure (mm Hg) & $6.75 \pm 2.5$ & $9.9 \pm 3.9$ & 0.23 \\
PAP systolic (mm Hg) & $35.6 \pm 10.7$ & $51 \pm 22.3$ & 0.27 \\
PAP diastolic (mm Hg) & $16.2 \pm 5.4$ & $22.7 \pm 14.9$ & 1 \\
Mean PAP (mm Hg) & $23.6 \pm 6.6$ & $33.7 \pm 16.6$ & 0.34 \\
PAOP (mm Hg) & $10.3 \pm 4.6$ & $13.3 \pm 3.9$ & 0.41 \\
TPG (mm Hg) & $13.4 \pm 9.3$ & $20.4 \pm 14$ & 0.43 \\
Qp/Os & $2.2 \pm 1.5$ & $3.1 \pm 1$ & 0.18
\end{tabular}

ABP, arterial blood pressure; HR, heart rate; NYHA, New York Heart Association (values from 1 to 4); PAOP, pulmonary arterial occlusion pressure; PAP, pulmonary arterial pressure; Qp/Qs, pulmonary-to-systemic flow ratio; RA, right atrium; TPG, transpulmonary gradient.

by Dotter et al. in $1949 .{ }^{14}$ Currently, the diagnosis of PAPVC is established with newer diagnostic modalities including transoesophageal echocardiography, CT angiography, MRI and pulmonary vein angiography during catheterization. ${ }^{15-20}$ A review of literature suggests that the most common type of PAPVC is right-sided pulmonary vein draining into the SVC or the RA. ${ }^{1,3}$ Alsoufi et al. ${ }^{3}$ found right-sided, left-sided and bilateral anomalous pulmonary veins in $91 \%, 7 \%$ and $2 \%$ of the patients with PAPVC. Similarly, we found right-sided anomalous pulmonary veins draining into the RA or SVC in the majority of our patients (71\%) (Fig. 1). Only four patients had left-sided anomalous pulmonary veins $(29 \%)$.

In our study, individuals were initially evaluated for several clinical conditions (most commonly dyspnoea, the presence of a murmur, arrhythmia or enlarged pulmonary vessels on a chest X-ray) and were found to have PAPVC. Only two patients in group I but four in group II were symptomatic (dyspnoea on exertion). The diagnosis was, in most cases, initially suspected by non-invasive studies, as previously documented in literature. ${ }^{16-19}$ In group I, the diagnosis was suggested by transthoracic echocardiography (TTE) in three patients (two patients had PFO and one had mildly dilated RV, conditions that were further evaluated), CT angiography of chest and cardiac MRI in two patients each (Table 1). In five patients of group II, the diagnosis was suspected during TTE (evidence of ASD). One patient was diagnosed with CT angiography of chest and the last one with a chest $\mathrm{X}$-ray, due to the abnormal position of a Hickman catheter.

The main strength of our study is that all patients underwent right heart catheterization for the evaluation of pulmonary hypertension, had oxygen saturation studies and when possible, angiography of the anomalous pulmonary veins. We found no statistical difference in mean PAP in group II as compared with

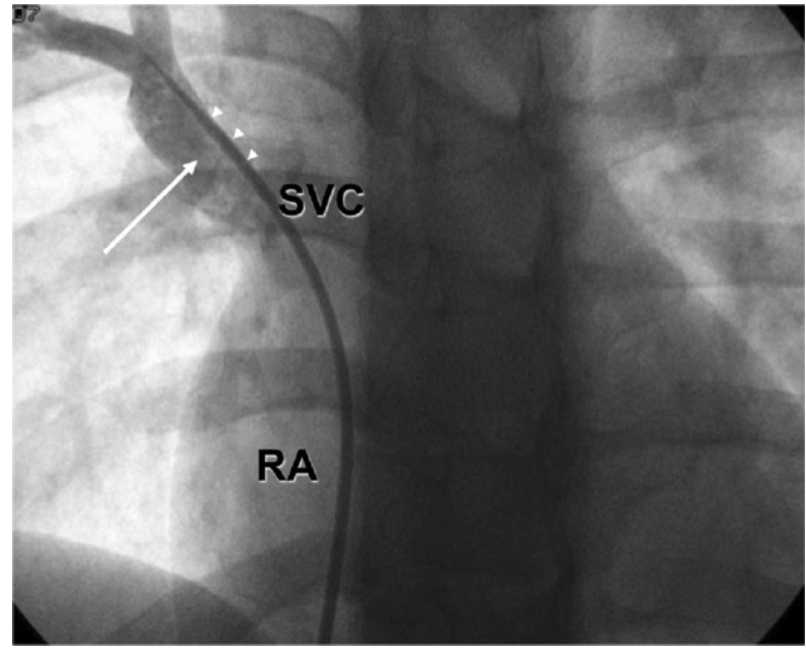

Figure 1 Fluroscopic image during the cardiac catheterization depicting right upper anomalous pulmonary vein (arrow) draining into the superior vena cava (SVC). RA, right atrium. Arrow heads mark the angiographic catheter entering the right upper anomalous pulmonary vein.

group I, possibly due to the small sample size of the study. Similarly, mean RA pressure was not significant between groups (Table 3). The development of PAH in PAPVC usually depends on the presence of associated congenital heart diseases such as ASD, the number of the anomalous pulmonary vein connections and the status of the pulmonary venous bed. ${ }^{21,22}$ The persistent high flow and pressure in the pulmonary arterial vasculature causes endothelial damage leading to loss of endothelial barrier function and imbalance of vasoactive mediators, favouring vasoconstriction, inflammation, thrombosis, cell proliferation, 
apoptosis and fibrosis, resulting in pulmonary vascular remodelling, irreversible $\mathrm{PAH}$ and right heart failure. $^{23,24}$

Recently, Majdalany et al. ${ }^{25}$ published their 20-year experience in patients with isolated PAPVC. These authors included 43 patients with PAPVC, 28 of these patients had RV overload and underwent corrective surgery. Twelve patients in this group were found to have elevated PAP before surgery, as estimated by the RVSP on transthoracic echocardiography. Of the 15 patients without RV overload, eight were found to have PAH. These authors observed an increase in PAP with increasing age. The mean estimated pressure in patients less than 40 years, 40-60 years and more than 60 years was 31, 38 and $51 \mathrm{~mm} \mathrm{Hg}$, respectively. These differences suggest that PAP increases with aging, posing the question whether PAPVC should be repaired in asymptomatic patients or not. Majdalany et $a l .{ }^{25}$ also documented a post-operative reduction in the estimated systolic PAP of $6 \mathrm{~mm} \mathrm{Hg}$ (range $1-17 \mathrm{~mm} \mathrm{Hg})$ in seven patients of the ones $(n=12)$ who had PAH preoperatively. In our study, we compared RVSP by Doppler echocardiography pre- and post-corrective surgery in six patients (all in group II). The RVSP was not statistically different before and immediately after corrective surgery; however the study was likely underpowered to detected any potential difference.

Congenital heart disease associated pulmonary hypertension is included in group I of the 4th World Symposium on Pulmonary Hypertension. ${ }^{11}$ Isolated anomalous PV return has been associated with PAH. ${ }^{8,26}$ Babb et al. ${ }^{8}$ and Saalouke et al. ${ }^{26}$ documented elevated PAP on cardiac catheterization in these patients. Saalouke et $a l .{ }^{26}$ suspected PAPVC on chest $\mathrm{X}$-ray and confirmed the diagnosis with cardiac catheterization and oximetry studies. They were the first to document the presence of PAH in isolated PAPVC as two of their five patients with this congenital heart condition had PAH with markedly increased pulmonary vascular resistance. Babb et al. ${ }^{8}$ diagnosed PAPVC on cardiac catheterization by oximetry studies. Two of their three patients with PAPVC were found to have PAH. Both patients presented with the complaints of exertional dyspnoea and signs of right heart failure. These initial studies supported the notion that PAPVC may be missed if not routinely investigated. According to autopsy studies, the prevalence is much higher $(0.4-0.7 \%)^{1,2}$ than the one observed in clinical practice. This could represent a large number of the patients with isolated PAPVC that remain asymptomatic for prolonged periods of time, even for life. It is possible that some of them may develop PAH during late adulthood, and this was not captured by our study.

Other limitations of our study include: (i) it is a retrospective review, which has its own limitations in terms of non-uniform patient evaluation and management; (ii) the small size of this cohort limited the power to detect potential differences in haemodynamic measurements between groups; (iii) it may not reflect the clinical characteristics of isolated PAPVC in the general population as there may be a significant number of patients who remain asymptomatic and thus are not diagnosed; (iv) group I had a lower mean PAP than the patients in the study by Majdalany et $a .^{25}$ This can be partly explained by differences in the age of the patients.

In spite of these limitations, our study included fairly large number of patients with PAPVC, keeping in mind the low prevalence of the disease. Patients were enrolled during the course of five years in an institution that has a busy adult congenital heart disease programme. Thus, an ideal prospective study would require the collaboration of multiple centres.

\section{CONCLUSION}

Patients with PAPVC had normal pulmonary artery pressure unless they have two pulmonary veins with anomalous return or associated conditions like sinus venosus ASD. Multi-centre prospective studies are needed to evaluate whether patients with isolated PAPVC develop PAH at long term.

\section{ACKNOWLEDGEMENT}

Dr Adriano Tonelli is supported by CTSA KL2 Grant \# RR024990 (A.R.T.) from the National Center for Research Resources (NCRR).

\section{REFERENCES}

1 Brody H. Drainage of pulmonary veins into the right side of the heart. Arch. Pathol. 1942; 33: 221-40.

2 Healey JF. An anatomic survey of anomalous pulmonary veins: their clinical significance. J. Thorac. Surg. 1952; 23: 433-4.

3 Alsoufi B, Cai S, Van Arsdell GS et al. Outcomes after surgical treatment of children with partial anomalous pulmonary venous connection. Ann. Thorac. Surg. 2007; 84: 2020-6.

4 Robicsek F, Daugherty HK, Cook JW et al. Sinus venosus type of atrial septal defect with partial anomalous pulmonary venous return. J. Thorac. Cardiovasc. Surg. 1979; 78: 559-62.

5 Brickner E, Hillis D, Lange RA. Congenital heart disease in adults. N. Engl. J. Med. 2000; 342: 256-63.

6 Al-Ahmari S, Chandrasekaran K, Brilakas E et al. Isolated partial anomalous pulmonary venous connection: diagnostic value of suprasternal color flow imaging and contrast echocardiography. J. Am. Soc. Echocardiogr. 2003; 16: 884-9.

7 Helseth HK, Peterson CR. Partial anomalous pulmonary venous drainage, intact atrial septum and acquired mitral stenosis. Surgical correction. Minn. Med. 1974; 57: 459-63.

8 Babb JD, McGlynn TJ, Pierce WS et al. Isolated partial anomalous venous connection: a congenital defect with late and serious complications. Ann. Thorac. Surg. 1981; 31: 540-1.

9 Al-Bustami O, Sharifi M, Hijazi ZM et al. Necessity for evaluation of anomalous pulmonary venous return before percutaneous closure of atrial septal defects—a case report. Angiology 2005; 56: 647-50.

10 Cragun DT, Lax D, Butman SM. Look before you close: atrial septal defect with undiagnosed partial anomalous pulmonary venous return. Catheter. Cardiovasc. Interv. 2005; 66: 432-5.

11 Hagen PT, Scholz DG, Edwards WD. Incidence and size of patent foramen ovale during the first 10 decades of life: an autopsy study of 965 normal hearts. Mayo Clin. Proc. 1984; Jan; 59(1): 17-20.

12 Simonneau G, Robbins IM, Beghetti M et al. Updated clinical classification of pulmonary hypertension. J. Am. Coll. Cardiol. 2009; 54: S43-54. 
13 McLaughlin VV, Archer SL, Badesch DB et al. ACCF/AHA 2009 expert consensus document on pulmonary hypertension: a report of the American College of Cardiology Foundation Task Force on Expert Consensus Documents and the American Heart Association: developed in collaboration with the American College of Chest Physicians, American Thoracic Society, Inc., and the Pulmonary Hypertension Association. Circulation 2009; 119: 2250-94.

14 Dotter CT, Hardisty NM, Steinberg I. Anomalous right pulmonary vein entering the inferior vena cava; two cases diagnosed during life by angiocardiography and cardiac catheterization. Am. J. Med. Sci. 1949; 218: 31-6.

15 Meissner I, Whisnant JP, Khandheria BK et al. Prevalence of potential risk factors for stroke assessed by transesophageal echocardiography and carotid ultrasonography: the SPARC study. Stroke prevention: assessment of risk in a community. Mayo Clin. Proc. 1999; 74: 862-9.

16 Kyo S. Color flow mapping 2-D Doppler in determining surgical indication for congenital heart disease. J. Cardiol. 1987; 15: S1115

17 Miller DS, Schwartz SL, Geggel RL et al. Detection of partial anomalous right pulmonary venous return with an intact atrial septum by transesophageal echocardiography. J. Am. Soc Echocardiogr. 1995; 8: 924-7.

18 Ammash NM, Seward JB, Warnes CA et al. Partial anomalous cenous connection: diagnosis by transesophageal echocardiography. J. Am. Coll. Cardiol. 1997; 29: 1351-8.
19 Otsuka M, Itoh A, Haze K. Sinus venosus type of atrial septal defect with partial anomalous pulmonary venous return evaluated by multislice CT. Heart 2004; 90: 901.

20 Festa P, Ait-Ali L, Cerillo AG et al. Magnetic resonance imaging is the diagnostic tool of choice in the preoperative evaluation of patients with partial anomalous pulmonary venous return. Int. J. Cardiovasc. Imaging 2006; 22: 685-93.

21 Webb GD, Smallhorn JF, Therrien J et al. Congenital heart disease. In: Braunwald E (ed.) Heart Disease: A Textbook of Cardiovascular Medicine, 8th edn. WB Saunders, Philadelphia, PA, 2008; 1561-624.

22 Geva T, Praagh SV. Anomalies of the pulmonary veins. In: Allan H, Shaddy R, Driscoll D et al. (eds) Moss and Adams' Heart Disease in Infants, Children and Adolescents, 7th edn. Williams \& Wilkins, Baltimore, MD, 2008; 761-91.

23 Rabinovitch M, Haworth SG, Castaneda AR et al. Lung biopsy in congenital heart disease: a morphometric approach to pulmonary vascular disease. Circulation 1978; 58: 1107-22.

24 Rabinovitch M. Pulmonary hypertension: pathophysiology as a basis for clinical decision making. J. Heart Lung Transplant. 1999; 18: 1041-53.

25 Majdalany DS, Phillips SD, Dearani JA et al. Isolated partial anomalous pulmonary venous connections in adults: twentyyear experience. Congenit. Heart Dis. 2010; 5: 537-45.

26 Saalouke MG, Shapiro SR, Perry LW et al. Isolated partial anomalous pulmonary venous drainage associated with pulmonary vascular obstructive disease. Am. J. Cardiol. 1977; 39: 439-44. 